\title{
Effect of NTN and Lmx1 $\alpha$ on the Notch Signaling Pathway during the Differentiation of Human Bone Marrow Mesenchymal Stem Cells into Dopaminergic Neuron-Like Cells
}

\author{
Jinhua Zhang $\mathbb{D},{ }^{1}$ Bo Yang $\left(\mathbb{D},{ }^{1}\right.$ Lilin Luo $\mathbb{D}^{1},{ }^{1}$ Linhui Li $\mathbb{D},{ }^{1}$ Xuantao Yang $\left(\mathbb{D},{ }^{1}\right.$ \\ Juanjuan Zhang $\mathbb{D}^{1},{ }^{1}$ Yuxin Xie $\mathbb{D}^{1},{ }^{1}$ Wanpu Wang $\mathbb{D}^{1},{ }^{1}$ and Shuaiyao Lu $\mathbb{D}^{2}$ \\ ${ }^{1}$ Department of Pathology, The First People's Hospital of Yunnan Province, \\ The Affiliated Hospital of Kunming University of Science and Technology, Kunming, China \\ ${ }^{2}$ Institute of Medical Biology, Chinese Academy of Medical Sciences and Peking Union Medical College, Kunming, China
}

Correspondence should be addressed to Wanpu Wang; 82898626@qq.com and Shuaiyao Lu; 342603482@qq.com

Received 26 November 2020; Revised 30 May 2021; Accepted 22 July 2021; Published 31 July 2021

Academic Editor: Giuseppina Martella

Copyright ( 2021 Jinhua Zhang et al. This is an open access article distributed under the Creative Commons Attribution License, which permits unrestricted use, distribution, and reproduction in any medium, provided the original work is properly cited.

Human bone marrow mesenchymal stem cells (h-BMSCs) have the potential to differentiate into dopaminergic neuron-like cells to treat Parkinson's disease. The Notch signaling pathway has been implicated in the regulation of cell fate decisions such as differentiation of BMSCs. This study investigated changes in the expression of Notch-related genes in the differentiation of BMSCs in vitro into dopaminergic (DA) neuron-like cells. BMSCs transfected with empty lentiviral vectors served as the control group and those transfected with NTN and $\operatorname{Lmx} 1 \alpha$ recombinant lentiviral vectors served as the experimental group. After induction and culture of NTN and Lmx $1 \alpha$-transfected h-BMSCs for 21 days, the cells exhibited features of dopaminergic neuron-like cells, which were observed by transmission and scanning electron microscopy and verified by immunofluorescence of tyrosine hydroxylase (TH) and dopamine transporter (DAT). These induced cells could secrete dopamine and had basic action potentials. Expression of the neural stem cell (NSC) markers, including octamer-binding protein (Oct4), paired box gene 6 (Pax6), and sex determining region Y-box 1 (SOX1), increased on day 14 of induction and decreased on day 21 of induction during differentiation. The human Notch signaling pathway PCR array showed a differential expression of Notch-related genes during the differentiation of h-BMSCs into DA neuron-like cells in vitro relative to that in the control group. In conclusion, h-BMSCs overexpressing NTN and Lmx $1 \alpha$ can successfully be induced to differentiate into dopaminergic neuron-like cells with a neuronal phenotype exhibiting fundamental biological functions in vitro, and NTN and Lmx1 $\alpha$ may affect the expression of Notch-related genes during differentiation.

\section{Introduction}

Parkinson's disease $(\mathrm{PD})$ is an age-related neurodegenerative disease that is clinically characterized by the death of dopaminergic (DA) neurons and the formation of Lewy bodies $[1,2]$. The mainstay of PD management is drug therapy, but drug therapy cannot prevent disease development. Fortunately, many studies have proved that bone marrow mesenchymal stem cells (BMSCs) are suitable for cell transplantation therapy in $\mathrm{PD}$ [3]. In our previous study, we demonstrated that DA can be efficiently induced from BMSCs overexpressing the LIM homeobox transcription factor $1 \alpha$
$(\operatorname{Lmx} 1 \alpha)$ and neurturin (NTN) [4]. When these BMSCs were grafted into 1-methyl-4-phenyl-1, 2, 3, 6-tetrahydro pyridine(MPTP-) lesioned right side substantia nigra of rhesus monkeys with $\mathrm{PD}$, they were found to improve impaired behavior [4]. Similarly, $\operatorname{Lmx} 1 \alpha$ has been shown to regulate midbrain DA neuronal differentiation, and direct injection of NTN into the midbrain restores degenerating DA neurons [5-9]. However, the exact mechanism by which BMSCs differentiate into DA neurons remains unclear.

Until recently, many reports studying the mechanism of differentiation of BMSCs have focused on signaling pathways such as the Notch, Wnt, and Sox signaling pathways. 
Notch family components play a considerable role in the regulation of cellular processes ranging from embryonic and postnatal development to adult stem cell differentiation [10-12]. Notch signal transduction mediates adjacent cellcell interactions. When the Notch receptor combined ligands, the intracellular Notch domain NICD is released from the membrane to activate the expression of transcription factors [13]. No further intermediate steps involving a second messenger and protein kinase are needed. Therefore, focusing on the Notch signaling pathway is the best way to study the mechanism of differentiation of BMSCs [14]. In the present study, we successfully established an h-BMSC cell line overexpressing $\operatorname{Lmx} 1 \alpha$ and NTN and attempted to investigate the role of Notch-related genes in the differentiation of BMSCs.

\section{Materials and Methods}

2.1. Cell Culture. Human BMSCs (h-BMSCs; HUXMA01001 cell line) were provided by Cyagen Biosciences (Guangzhou, China) and cultured in growth medium for human BMSCs (Cyagen Biosciences, Guangzhou, China) in air containing $5 \% \mathrm{CO}_{2}$ at $37^{\circ} \mathrm{C}$. The cells were cultured to $90 \%$ confluence, digested with trypsin, and divided into two flasks.

2.2. Gene Transfection of $h$-BMSCs. Recombinant $\operatorname{Lmx} 1 \alpha$ and NTN plasmids were constructed using lentivirus particles and established by Shanghai Taitool Bioscience Co., Ltd., and the recombinant plasmid containing both these genes was designated PLV-duaI-Lmx $1 \alpha$-NTN. h-BMSCs were seeded into the wells of six-well plates at a concentration of $1 \times 10^{5}$ per well, after which the cells were infected with the recombinant and control lentiviruses. The multiplicity of infection (MOI) was set at 10, 50, and 100, and the duration was set at 48 hours to explore maximal transfection efficiency.

2.3. Induction of DA from $h$-BMSCs. Human BMSCs were seeded into six-well plates at a density of $1 \times 10^{5}$ per well in the growth medium. Twenty-four hours later, cultured h-BMSCs were transfected with $\operatorname{Lmxl} \alpha$ and NTN recombinant lentivirus and cultured in maintenance medium (low-glucose DMEM/F12 with 5\% fetal bovine serum and $1 \%$ antibiotics). After one day, the medium was changed to the DMEM/F12 medium with $20 \%$ fetal bovine serum, $6 \mathrm{~mm} \beta$-mercaptoethanol, and $20 \mathrm{ng} / \mathrm{mL} \beta$-fibroblast growth factor. At $24 \mathrm{~h}$ after incubation, in the preinduction medium, h-BMSCs were induced in the induction medium (DMEM/ F12 medium containing 15\% fetal bovine serum, $6 \mathrm{~mm}$ $\beta$-mercaptoethanol, $20 \mathrm{ng} / \mathrm{mL} \beta$-fibroblast growth factor, $1.7 \mathrm{~m}$ recombinant human Sonic hedgehog factor, $100 \mathrm{ng} /$ $\mathrm{mL}$ fibroblast growth factor 8 , and $0.3 \mathrm{~g} / \mathrm{mL}$ retinoic acid). The medium was replaced with fresh induction medium every 4-5 days. Fourteen days later, the medium was changed to the differentiation maintenance medium (DMEM/F12 with $2 \% \mathrm{~B} 27$ and $1 \% \mathrm{~N}_{2}$ ).
2.4. Western Blotting. h-BMSCs were transfected with Lmx $1 \alpha$ and NTN recombinant lentivirus for $48 \mathrm{~h}$ and then incubated with $300 \mu \mathrm{L}$ of RIPA buffer containing $1 \%$ protease inhibitor for $10 \mathrm{~min}$ at $4^{\circ} \mathrm{C}$. The resultant lysates were centrifuged at $12000 \mathrm{rpm}$ for $10 \mathrm{~min}$, and the supernatant was collected and used to determine the protein concentration by BCA assay, according to the instructions of the BCA protein assay kit. Then, $80 \mu \mathrm{g}$ samples of total protein were separated by electrophoresis in electrophoresis buffer (3.03 g Tris, $18.77 \mathrm{~g}$ glycine, and $0.1 \% \mathrm{SDS}$ ) at $60 \mathrm{~V}$ for $25 \mathrm{~min}$ and $90 \mathrm{~V}$ for $3 \mathrm{~h}$ and then transferred to polyvinylidene difluoride (PVDF) membranes at $200 \mathrm{Ma}$ for $1 \mathrm{~h}$. The membranes were blocked in 5\% bovine serum albumin for $1 \mathrm{~h}$ and incubated with primary antibodies against Lmx1 $\alpha$ (1:1000, ab139726, Abcam), NTN (1:500, ab8061, Abcam), and glyceraldehyde-3-phosphate dehydrogenase (GAPDH, $1: 1000$, P30008, Abmart) in Tris-buffered saline (TBS) overnight at $4^{\circ} \mathrm{C}$. The membranes were then incubated with secondary antibodies $(1: 2000,7074$, Cell Signaling Technology) at room temperature for $2 \mathrm{~h}$. The membranes were then analyzed with enhanced chemiluminescence reagent (Biyuntian, China).

2.5. Real-Time Quantitative RT-PCR. After transfection for $48 \mathrm{~h}$, total RNA was extracted using TRIzol $^{\text {TM }}$ Reagent (Lifetech, Thermo Fisher Scientific, Inc.) according to the manufacturer's protocol. Before RNA was reverse-transcribed into cDNA using RevertAid ${ }^{\mathrm{TM}}$ First Strand cDNA Synthesis Kit (Fermentas, Canada), the RNA concentration was measured by NanoDrop ND1000 (ThermoFisher, USA). The primers were designed with Beacon Designer 7.90. Table 1 provides the primers used in this study. Target genes were obtained by qPCR using ABI StepOne, and a PCR reaction system was established as follows: $5 \mu \mathrm{L}$ of $2 \times \mathrm{SYBR}$ Green Master Mix, $0.25 \mu \mathrm{L}$ of forward primer, $0.25 \mu \mathrm{L}$ of reverse primer, $0.2 \mu \mathrm{L}$ of ROX, $0.5 \mu \mathrm{L}$ of cDNA template, and $3.8 \mu \mathrm{L}$ of nuclease-free water. The PCR conditions were as follows: $2 \mathrm{~min}$ at $50^{\circ} \mathrm{C}$; followed by 40 cycles of $10 \mathrm{~min}$ at $95^{\circ} \mathrm{C}, 15 \mathrm{~s}$ at $95^{\circ} \mathrm{C}$, and $30 \mathrm{~s}$ at $60^{\circ} \mathrm{C}$; and $15 \mathrm{~s}$ at $95^{\circ} \mathrm{C}, 30 \mathrm{~s}$ at $60^{\circ} \mathrm{C}$, and $15 \mathrm{~s}$ at $95^{\circ} \mathrm{C}$. The relative quantities of NTN and $\operatorname{Lmx} 1 \alpha$ mRNA were normalized to that of GAPDH mRNA.

2.6. Immunofluorescence. After fixing with paraformaldehyde for $30 \mathrm{~min}$, the cells were blocked with $5 \%$ sheep serum for $60 \mathrm{~min}$ before permeation in $0.2 \%$ Triton X-100 for $20 \mathrm{~min}$. Then, the cells were incubated with primary antibodies overnight at $4^{\circ} \mathrm{C}$ : tyrosine hydroxylase $(\mathrm{TH} ; 1: 200$, ab75875, Abcam) and dopamine transporter (DAT; $1: 400$ dilution, Sc-32259, Santa). After the cells were washed four times with $0.01 \mathrm{~mol} / \mathrm{L}$ of phosphate-buffered saline-Tween (PBST) for $5 \mathrm{~min}$ each, they were incubated at room temperature for $2 \mathrm{~h}$ with fluorescence-labeled secondary antibodies: anti-rabbit IgG (1:1000, 072-01-15-06, KPL) and anti-mouse $\operatorname{lgG}$ (1:1000 dilution, 072-03-18-06, KPL). The antibodies were rinsed again with PBST, and the slides were sealed with $50 \mu \mathrm{L}$ of 4, 6-diamidino-2-phenylindole (DAPI). The slides were photographed with an ortho-fluorescence microscope. 
TABle 1: Primer sequences of NTN and $\operatorname{Lmx} 1 \alpha$.

\begin{tabular}{lc}
\hline Gene & Primer sequence $\left(5^{\prime}-3^{\prime}\right)$ \\
\hline NTN & AAAGATGGCAGGAGAGAT (forward) \\
& ACCAGGACATATGAATTACTAC (reverse) \\
Lmxl $\alpha$ & CTTGTGAACGAAGATAAGTG (forward) \\
& TCTACCTCTGAAGCATCC (reverse) \\
GAPDH & AAAGGGTCATCATCTCTG (forward) \\
& GCTGTTGTCATACTTCTC (reverse) \\
\hline
\end{tabular}

NTN, neurturin; Lmx1 $\alpha$, LIM homeobox transcription factor $1 \alpha$; GAPDH, glyceraldehyde-3-phosphate dehydrogenase.

2.7. Flow Cytometry. The cells were digested by $0.25 \%$ trypsin, collected, and resuspended with $1 \mathrm{~mL}$ of $4 \%$ paraformaldehyde for $3 \mathrm{~min}$. The cells were resuspended in PBS containing $0.1 \%$ Triton X-100 and permeabilized for $30 \mathrm{~min}$. The collected cells were then sealed with $5 \%$ sheep serum at $37^{\circ} \mathrm{C}$ for $30 \mathrm{~min}$, and the supernatant was discarded after centrifugation. The cells were resuspended with primary antibodies against sex determining region Y-box 1 (SOX1; ab109290, Abcam), paired box gene 6 (Pax6; ab5790, Abcam), and octamer-binding protein (Oct4; ab181557, Abcam) at a 1:500 dilution with $3 \%$ goat serum at $37^{\circ} \mathrm{C}$ for $1 \mathrm{~h}$. The cells were then treated with secondary antibodies diluted to $1: 1000$ using $3 \%$ goat serum for $30 \mathrm{~min}$, after which the fluorescence of the PBS-resuspended cells was measured by flow cytometry.

2.8. ELISA. To assess the function of Lmxl and NTN overexpression, ELISA was performed to compare the dopamine levels in the control and transfected groups (Cusabio, Wuhan, China). After h-BMSCs were transfected with NTN and $\operatorname{Lmx} 1 \alpha$ recombinant lentiviruses, protein was collected on day 21 and stored at $-80^{\circ} \mathrm{C}$ for the above test.

2.9. Whole-Cell Patch-Clamp Recordings. Cells were rested for $30 \mathrm{~min}$, perfused $(2 \mathrm{~mL} / \mathrm{min})$ with extracellular fluid $\left(140 \mathrm{~mm} \mathrm{NaCl}, 5.4 \mathrm{~mm} \mathrm{KCl}, 1.8 \mathrm{~mm} \mathrm{CaCl}_{2}, 1 \mathrm{mM} \mathrm{MgCl}\right.$, $10 \mathrm{~mm} \quad \mathrm{~N}$-2-hydroxyethylpiperazine- $\mathrm{N}$-2' -ethanesulfonic acid (HEPES), and $11.1 \mathrm{~mm}$ glucose dissolved in $800 \mathrm{~mL}$ of ultrapure water with the $\mathrm{pH}$ adjusted to 7.4 with $\mathrm{NaOH}$ ) to remove the surrounding impurities. The microelectrode was pushed into the cell at $45^{\circ}$, and the change in microelectrode resistance is detected at all times during the process. When the electrode comes into contact with the cell, the positive pressure is relieved and stabilized for 5-10 s. Once the seal resistance was higher than $1 G \Omega$, the cell was given fast capacitance compensation, and after 2 min of stabilization, a closed connection is formed. Then, we disrupted the cell membrane and obtained whole-cell recordings.

2.10. Human Notch Signaling Pathway PCR Array. RT ${ }^{2}$ profiler $^{\mathrm{TM}}$ PCR array of the human Notch signaling pathway was purchased from SA Biosciences. Total RNA was isolated from cells using TRIzol (Invitrogen). RNA samples were purified using the RNeasy ${ }^{\mathrm{TM}}$ MinElute Kit (Qiagen). SuperScript III Reverse Transcriptase (Invitrogen) was used for cDNA synthesis. The cDNAs were used for $\mathrm{RT}^{2}$ profiler PCR array with $\mathrm{RT}^{2}$ SYBR Green ROX qPCR Master Mix (Qiagen). Fold change in the mRNA expression levels between groups was calculated by $2^{-\Delta \Delta \mathrm{Ct}}$.

2.11. Statistical Analysis. Statistical analyses were conducted using IBM SPSS Statistics (version 18) and GraphPad Prism (version 8). A two-tailed Student's $t$ test was used to test for statistical differences in group means between the control and overexpression groups. $P$ values of $<0.05$ were considered statistically significant.

\section{Results}

3.1. Transfection Efficiency of $L m x 1 \alpha$ and NTN Recombinant Lentivirus. When h-BMSCs were infected with NTN and $\operatorname{Lmx} 1 \alpha$ recombinant lentiviruses for 48 hours, protein expression was the most abundant when MOI was 50 (Figure 1(a)). Western blot, grayscale analysis, and PCR demonstrated that the NTN and Lmx1 $\alpha$ expression levels significantly differed between the transfection and control groups ( $p<0.005$; Figures $1(b)-1(d)$ ). These results confirm the successful transfection and efficient expression of the NTN and Lmxl $\alpha$ in h-BMSCs.

\subsection{Identification of $h$-BMSC-Derived Dopaminergic} Neurons. The results of the scanning electron microscopy revealed that most of the neurons in the induction group appeared different compared to those in the control group. The shape of the neurons changed after 21 days of induction and could be visualized as strong stereoscopic appearance, synaptic connections, and neural network; no similar change in shape was observed in the controls (Figure 2(a)). Transmission electron microscopy showed clear nuclei, lobed nucleoids, and visible microvilli in the control group, while decreased organelles after 14 days of induction (Figure 2(b)). After 21 days of induction, inconspicuous nuclei and relatively large numbers of mitochondria were observed (Figure 2(b)). These morphological features suggested that h-BMSCs of the control group are metabolically active and exhibited poor differentiation and that neuronal differentiation was induced in the PLV-duaI-Lmx $1 \alpha$-NTNinfected h-BMSCs.

$\mathrm{TH}$ and DAT immunofluorescence were performed to verify whether NTN and $\operatorname{Lmx} 1 \alpha$ overexpressions could promote the differentiation of dopaminergic neurons from h-BMSCs. As shown in Figure 3, TH and DAT were overexpressed in h-BMSCs after 14 and 21 days of induction, respectively, compared to the corresponding levels in the control group. Dopamine expression was significantly increased in the transfection group relative to the control group after 21 days of incubation (Figure 2(c)).

The action potential was detected and recorded using a whole cell membrane clamp technique using a setting of $-60 \mathrm{mV}$ and an electrode impedance of $2-5 \mathrm{M} \Omega$. The amplitude of the control group is almost flat throughout the frequency (Figure 4(a)). In contrast, the induced group of neurons showed an increase in amplitude, presenting a typical neuron waveform (Figure 4(b)). Together, these 


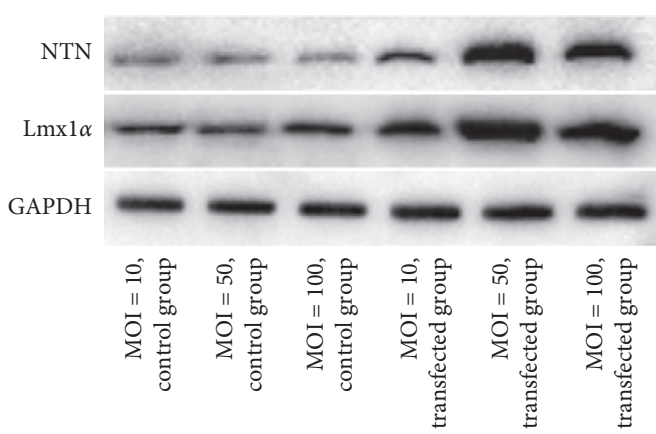

(a)

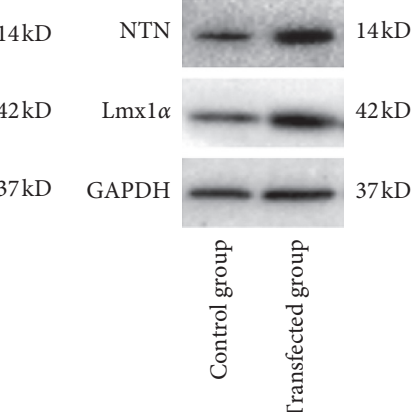

(b)
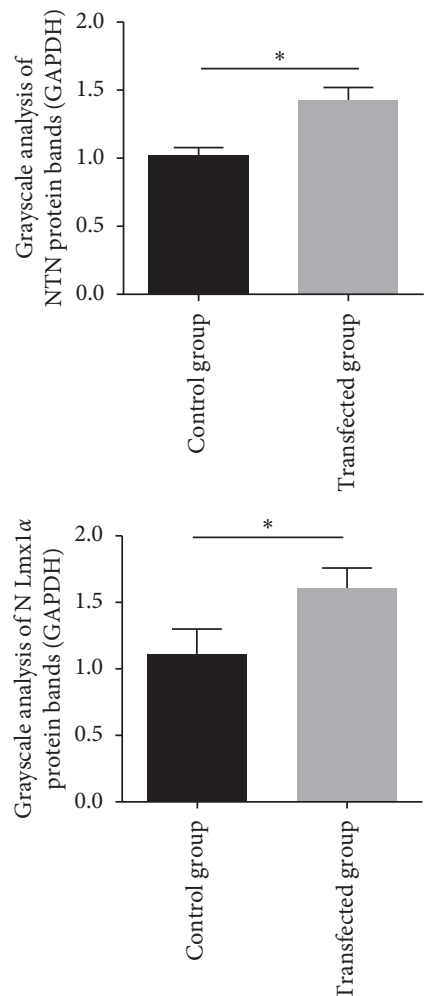

(c)
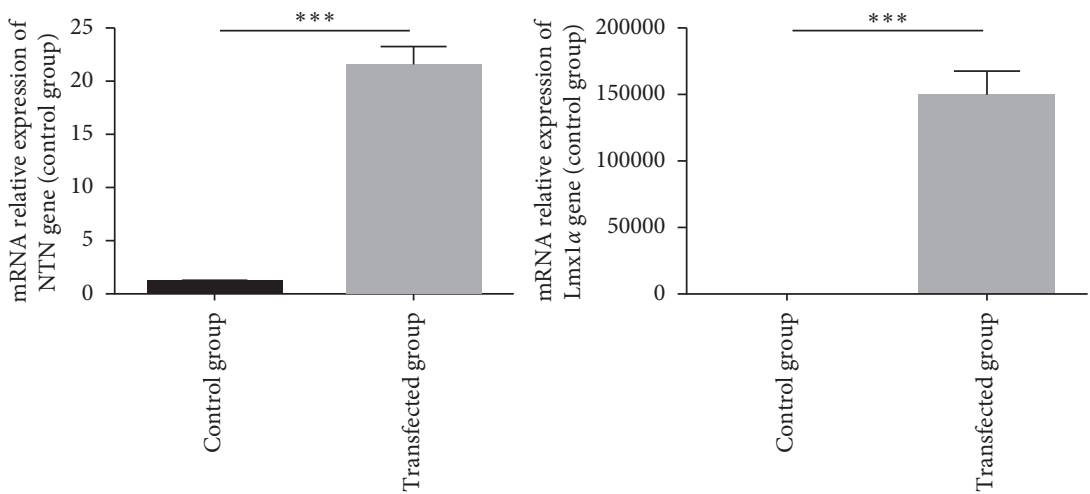

(d)

FIgURE 1: Gene transfection adopted by recombinant lentivirus to overexpress NTN and Lmx1 $\alpha$. (a) Protein expression was the most abundant when MOI was 50. (b)-(d) Western blot analysis, grayscale analysis, and PCR analysis verified significantly high expression of NTN and $\operatorname{Lmx} 1 \alpha$ in the transfected group relative to the control group. GAPDH was used as an internal control. NTN, neurturin; Lmx1 $\alpha$, LIM homeobox transcription factor $1 \alpha$; GAPDH, glyceraldehyde-3-phosphate dehydrogenase; MOI, multiplicity of infection. ${ }^{*} P<0.05$. ${ }^{* * *} P<0.001$. 


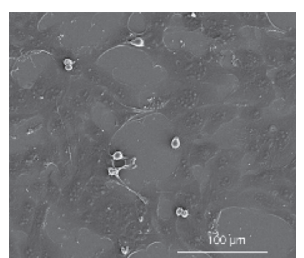

Control group

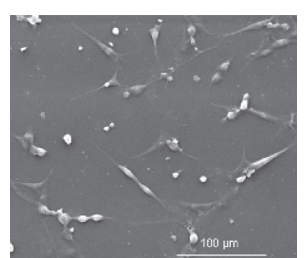

Transfected group

(a)

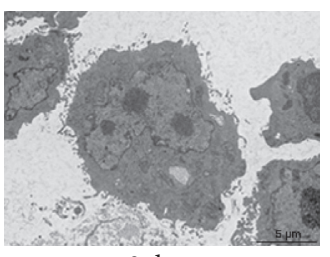

0 days

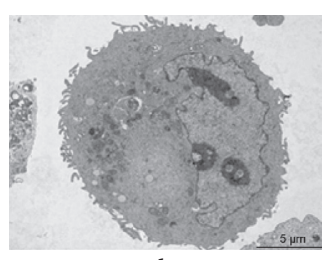

7 days

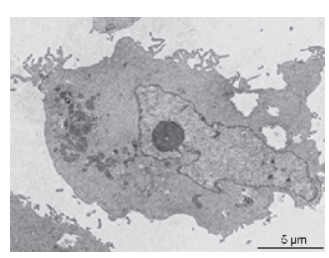

14 days

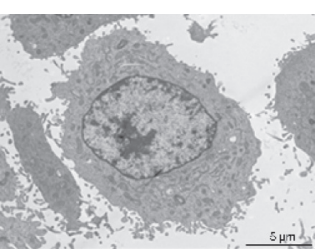

21 days

(b)

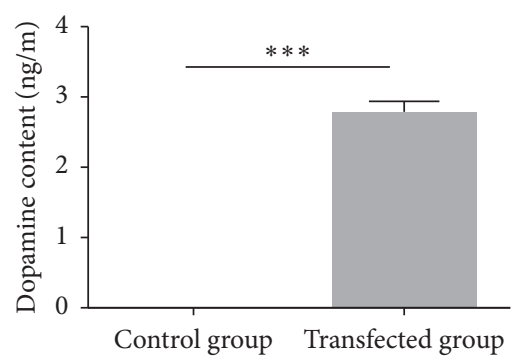

(c)

FIGURE 2: Identification of dopaminergic neurons differentiated from h-BMSCs. (a) Scanning electron microscopy showing ultrastructural characteristics of the transfection group changed after 21 days of induction. Scale bar $=100 \mu \mathrm{m}$. (b) Transmission electron microscopy results showing ultrastructural characteristics of the transfection group changed after 7, 14, and 21 days. Scale bar $=5 \mu \mathrm{m}$. (c) Dopamine expression of the transfection group significantly increased relative to the control group after 21 days of incubation. ${ }^{* * *} P<0.001$.

findings indicated the capacity of DA neurons to generate action potentials.

\subsection{Intermediate Process of Differentiation of h-BMSCs into} DA Neuron-Like Cells. Flow cytometry revealed that Oct4 expression increased at 14 days of incubation and gradually decreased again at 21 days of incubation. The expression levels of Pax6 and SOX1 were similar to that of Oct4 (Figure 5), indicating that h-BMSCs do not directly differentiate into DA, but are subject to an intermediate process of forming the neural stem cell- (NSC-) like cells.

3.4. Regulation of NTN and $L m x 1 \alpha$ in the Differentiation of $h$-BMSCs into DA. We performed $\mathrm{RT}^{2}$ Profiler ${ }^{\mathrm{TM}} \mathrm{PCR}$ array to further explore the mechanisms of the Notch signal pathway in the differentiation of h-BMSCs into DA. There was a significant difference in expression of related genes between the control and induction groups at 7, 14, and 21 days (Figures 6(a) and 6(b)). The correlation in Notch-related genes between the control and induction groups at 7 , 14 , and 21 days was examined using a scatter plot (Figure 6(c)). Figure 6(d) and Table 2 illustrate the differential expression of genes between the two groups. These genes include genes involved in intercellular communication, cell development, and differentiation, suggesting that NTN and Lmx $1 \alpha$ most likely play a role in differentiation through the indirect regulation of the Notch signaling pathway.

\section{Discussion}

PD is clinically characterized by the death of DA neurons. The treatment of PD includes drug therapy and operative management, which cannot prevent disease development. BMSCs have the ability of preventing immune rejection and secreting abundant trophic factors. BMSCs have been the focus of research to develop cell-based therapeutic strategies for PD. Recent progress in cell-based therapeutic strategies for PD is usually studied in two aspects. One is to promote the differentiation of MSCs into dopaminergic neurons and the other is to protect dopaminergic neurons. NTN is a neurotrophic factor that acts specifically on DA and motor neurons in the midbrain $[5,15,16]$. Lmxl $\alpha$ regulates all the stages of dopaminergic neuronal differentiation $[7,8,17,18]$. Overexpression of NTN and $\operatorname{Lmx} 1 \alpha$ in rhesus BMSCs has been shown to strongly promote DA neurogenesis in vitro and restore motor function after transplantation in vivo in a PD monkey model, established by treating monkeys with 1-methyl-4-phenyl-1, 2, 3, 6- 

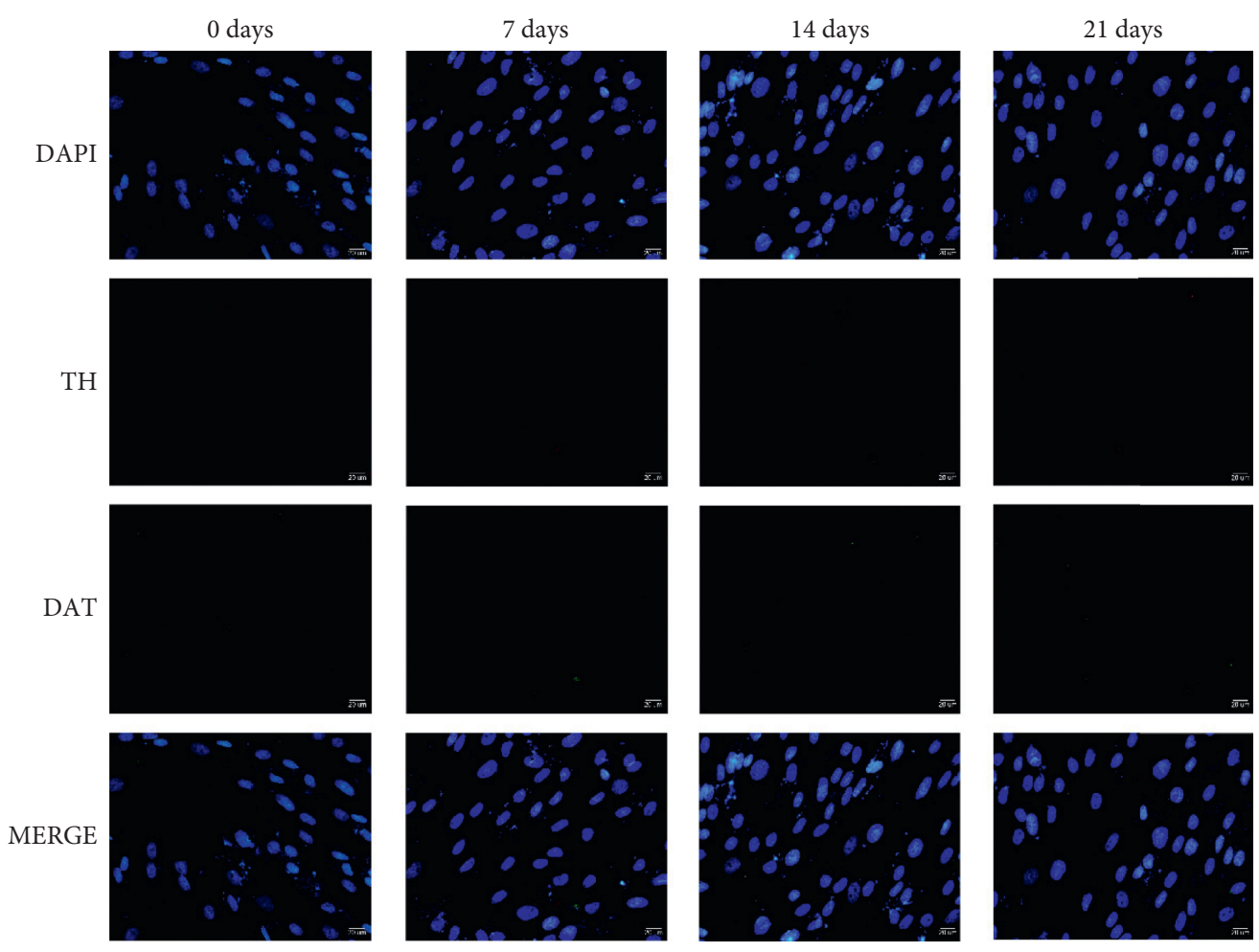

(a)
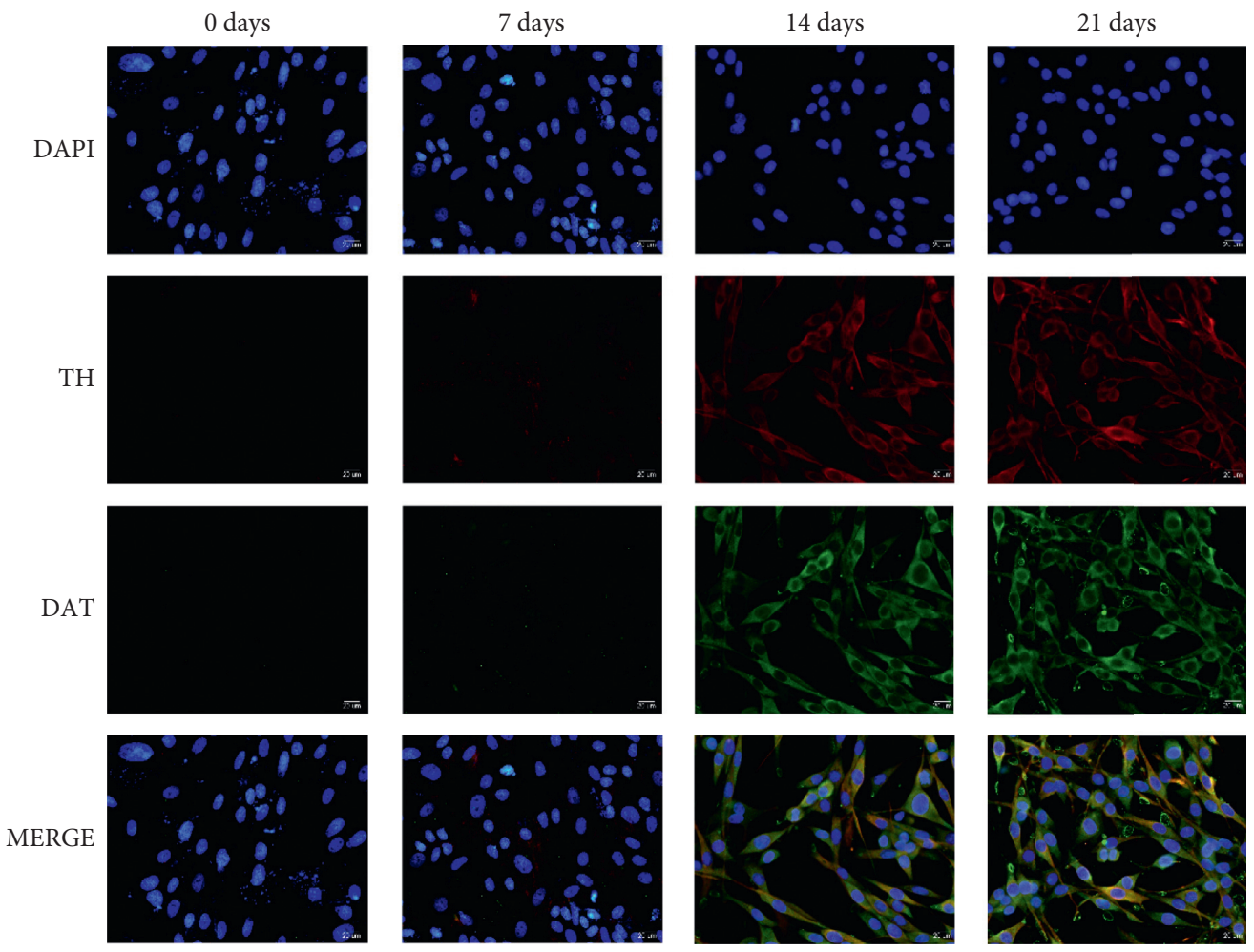

(b)

FIGURE 3: Immunofluorescence analysis showing TH (red fluorescence) and DAT (green fluorescence) expression of the transfection group was increased after 14 and 21 days of induction compared to the corresponding levels in the control group. Scale bar $=20 \mu \mathrm{m}$. TH, tyrosine hydroxylase; DAT, dopamine transporter. 


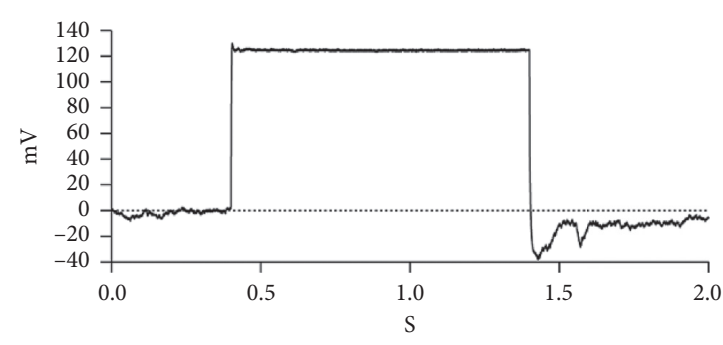

(a)

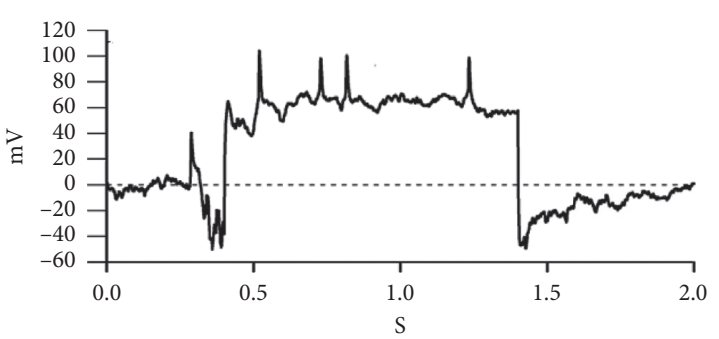

(b)

Figure 4: Whole cell membrane clamp recordings showing the electrophysiological behavior of the transfected group (b) presenting neuron waveform after 21 days of induction, compared to the control group (a).

tetrahydropyridin [4]. However, the mechanism by which BMSCs differentiate into DA neurons is unclear.

In present study, we chose the clinically widely used lentiviral vectors. Lentiviral vectors are characterized by stable gene expression and higher transgene payloads [19]. The focus of h-BMSCs transplantation for PD is the directed differentiation of h-BMSCs into dopaminergic neurons. The cells after induction exhibited ultrastructural characteristics of dopaminergic neuron-like cells by transmission and scanning electron microscopy. These induced cells could express TH and DAT. It is important to generate an action potential for the functional qualification of neurons. The expression of dopamine was significantly increased in the transfected group after 21 days of incubation. This means that the induced h-BMSCs are biologically able to secrete dopamine. In addition, we used patch-clamp recordings to show that the h-BMSC-derived DA neuron-like cells were capable of generating action potentials in the genetically modified h-BMSCs at 21 days of incubation, which indicates that nerve cells transmit excitement.

Ma et al. reported that NSC-like cells can be efficiently produced from h-BMSCs under appropriate culture conditions [20]. In addition, these NSC-like cells retained their capability to express molecular markers of neurons. In our study, to explore whether the differentiation of h-BMSCs to DA neurons involves neural stem cells (NSCs), we detected the expression of the NSC markers Pax6, SOX1, and Oct4. Flow cytometry analysis revealed that Pax6, SOX1, and Oct4 expression levels were elevated after 14 days in the transfection group, although these levels decreased at 21 days compared to that at 14 days. These results confirm that BMSCs differentiate into DA neurons through a process of NSC-like cells.

NTN is characterized by its capacity to nourish, protect, and repair damage to DA neurons. $\operatorname{Lmx} 1 \alpha$ is a crucial factor in the development of dopaminergic neurons. However, the mechanism by which BMSCs differentiate into DA neurons is unclear. Multiple signaling pathways are involved in the differentiation of h-BMSCs into dopaminergic neurons, including $\mathrm{SHH}$, Wnt, and Notch signaling pathway. No further intermediate steps involving a second messenger and protein kinase are needed during the transmission of the Notch signaling pathway. Currently, the mechanisms involved in the differentiation of BMSCs are focused on the Notch signaling pathway [21, 22]. The Notch pathway controls processes such as neurogenesis and neural stem cell maintenance $[23,24]$. Therefore, the human Notch signaling pathway PCR array was performed to detect the differential expression of Notch-related genes. The Notch signaling pathway PCR array contains 84 genes, including Notch signaling pathway ligands, receptors, target genes, cell proliferation and differentiation-related genes, and neurogenesis-related genes. In addition, this array also contains other signaling pathways that intersect with the Notch signaling pathway, such as Sonic hedgehog and Wnt receptor signaling pathway member.

The five Notch ligands found in mammals are Jagged1, Jagged2, Delta1, Delta3, and Delta4 [25]. Presenilin2 (PSEN2) and ADAM metallopeptidase domain 10 (ADAM10) participate in an essential role in the proteolytic release of NICD from the Notch receptor. At 7 days of induction, the expression of Jagged1 and Presenilin2 was significantly reduced compared with the control, which showed that the Notch signaling pathway was inhibited during induction differentiation. At 14 days of induction, similar results were obtained for Jagged1, Jagged2, ADAM10, and PSEN2 expression. HES1 expression was reduced compared with that in the control group at 14 days of induction. The low expression of PSEN2 further downregulates the target genes of Notch, suggesting that multiple mechanisms are involved in the suppression of the Notch signaling pathway. These observations indicate that the expression levels of Notch signal molecules are suppressed when h-BMSCs are induced to differentiate into neural cells. However, the Notch signaling pathway is a multistep and multifactorial process.

During the differentiation of BMSCs into DA neuronlike cells, the expression of genes related to neurogenesis was changed, compared with the control. For instance, the expression of FBJ murine osteosarcoma viral oncogene homolog (FOS) was significantly increased at 7 days of induction. Fos plays an important role in neurogenesis [26]. Paired box 5 (PAX5) expression was increased at 14 days of induction, relative to the control. PAX5 acts in cell fate specification and proliferation of neural precursor cells during development $[27,28]$. The expression of genes related to Wnt and SHH signaling components was also changed. The expression of frizzled family receptor 7 (FZD7) was increased and smoothened (SMO) decreased at 14 days of induction. To fully understand the role of Notch in the differentiation of h-BMSCs into neural cells, more in-depth studies are needed, especially at the molecular level. 

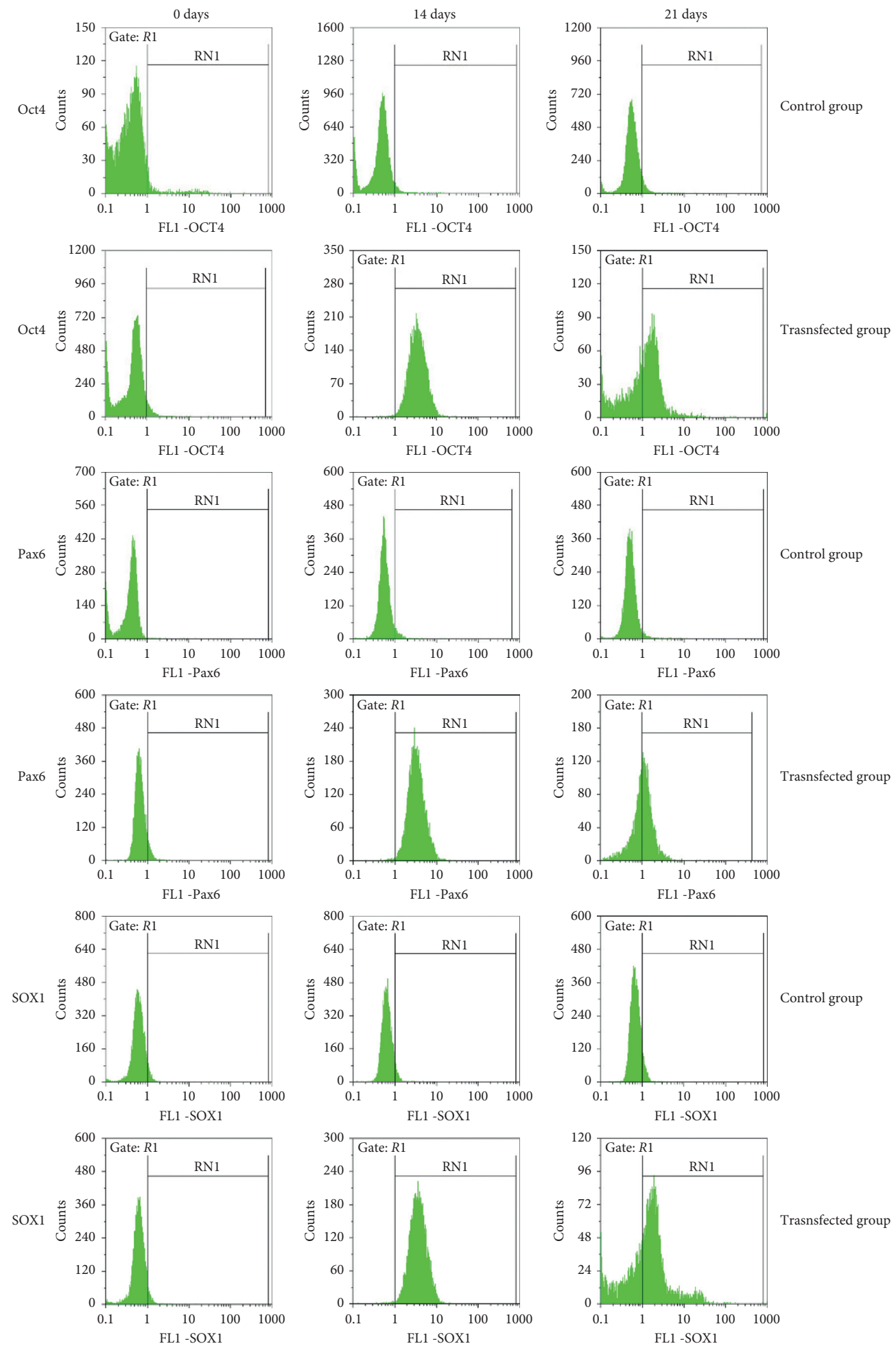

FigURE 5: Flow cytometry revealing Oct4, Pax6, and SOX1 expressions of NSC markers increased at 14 days of incubation and gradually decreased again at 21 days of incubation. Oct4, octamer-binding protein; Pax6, paired box gene 6; SOX1, sex determining region Y-box 1; NSC, neural stem cell. 


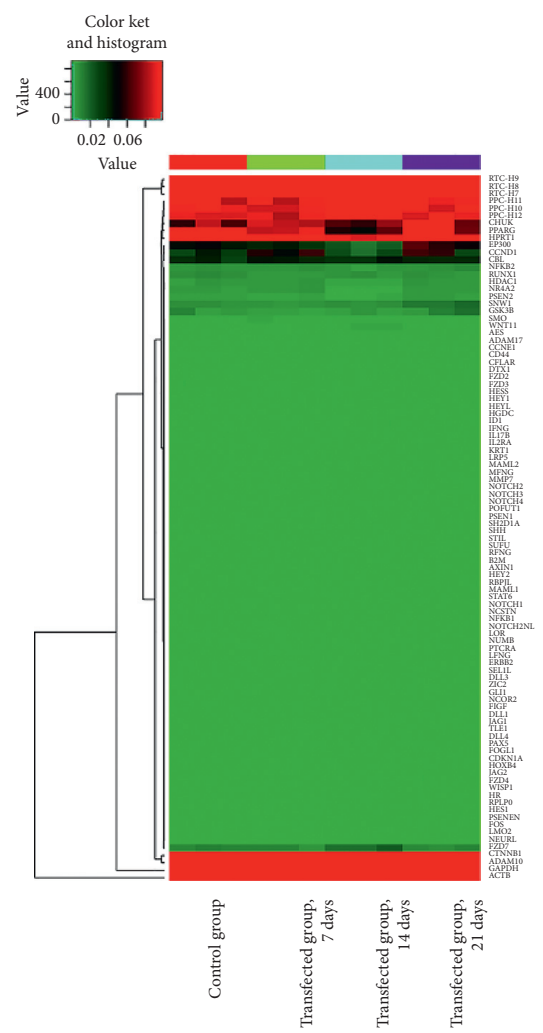

(a)
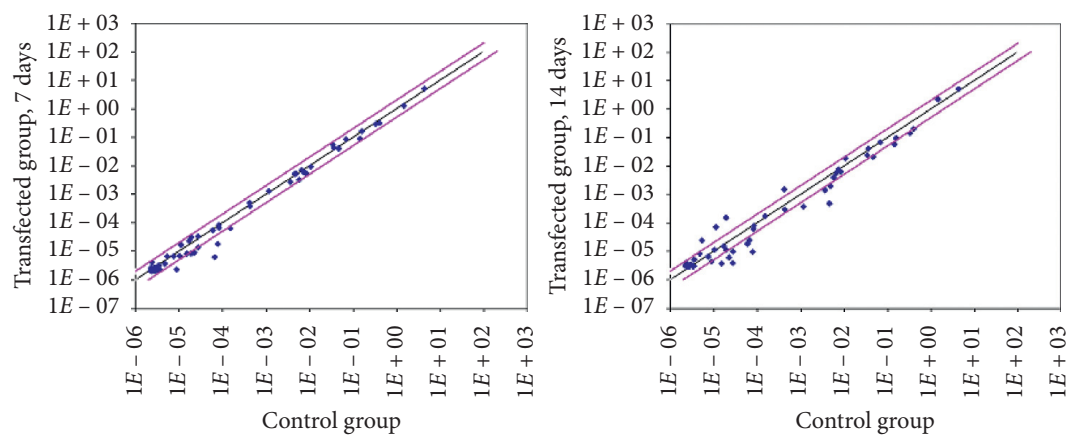

(c)

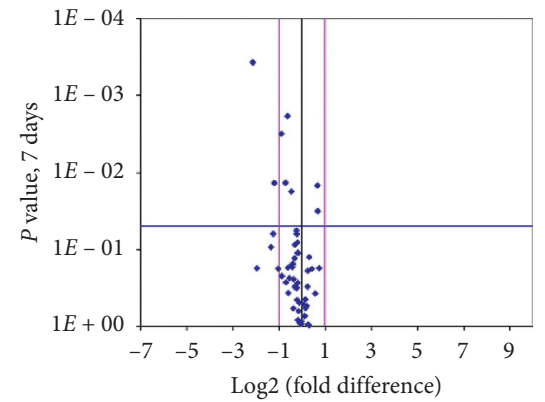

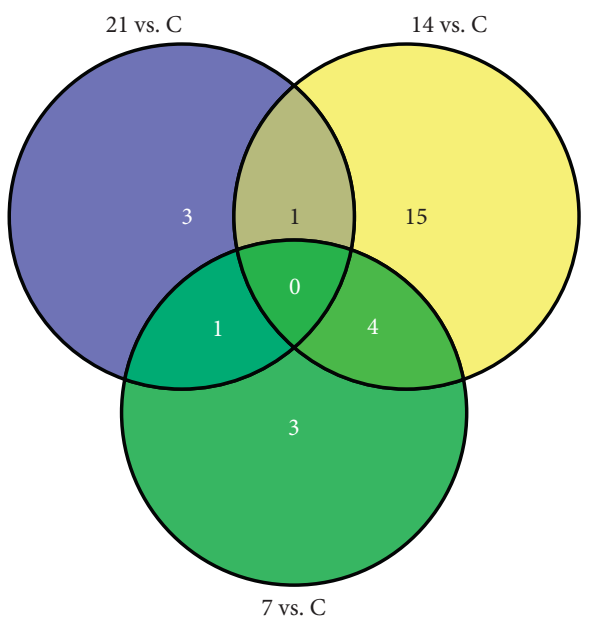

(b)
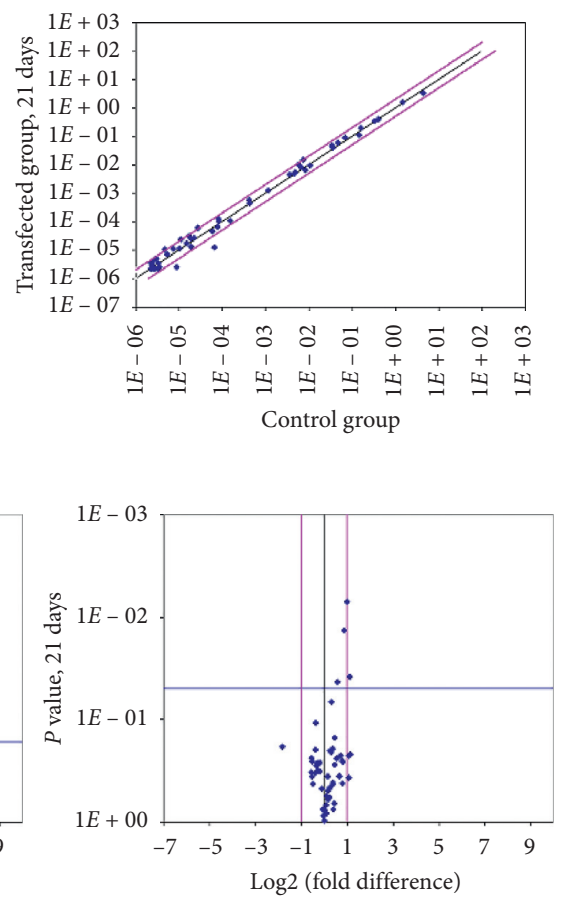

(d)

FIGURE 6: Differently expressed Notch-related genes between the control and transfected groups. (a) Heat maps showing significant changes in the expression of Notch-related genes between the control and transfected groups. (b) Venn diagram based on the differential expression of Notch-related genes between the two groups. (c) Scatter plots showing the differentially expressed Notch-related genes in the control group and transfection group. (d) Volcanic diagram showing the number of differentially expressed Notch-related genes. 
TABLE 2: Notch pathway qPCR results during DA neuronal differentiation.

\begin{tabular}{|c|c|c|c|c|c|c|c|}
\hline Gene symbol & Group & Regulation & $P$ value & Gene symbol & Group & Regulation & $P$ value \\
\hline CCND1 & 7 vs. $\mathrm{C}$ & $\mathrm{Up}$ & 0.032 & NCSTN & 14 vs. C & $\mathrm{Up}$ & 0.024 \\
\hline FOS & 7 vs. C & $\mathrm{Up}$ & 0.015 & NEURL & 14 vs. C & Down & 0.044 \\
\hline JAG1 & 7 vs. $\mathrm{C}$ & Down & 0.014 & NR4A2 & 14 vs. $\mathrm{C}$ & Down & 0.001 \\
\hline PPARG & 7 vs. C & Down & 0.014 & PAX5 & 14 vs. C & $\mathrm{Up}$ & 0.035 \\
\hline PSEN2 & 7 vs. $\mathrm{C}$ & Down & 0.018 & PPARG & 14 vs. C & Down & 0.004 \\
\hline RUNX1 & 7 vs. C & Down & 0.002 & PSEN2 & 14 vs. C & Down & 0.001 \\
\hline TLE1 & 7 vs. C & Down & 0.003 & SMO & 14 vs. C & Down & 0.003 \\
\hline RPLP0 & 7 vs. C & Down & 0.032 & TLE1 & 14 vs. C & Down & 0.001 \\
\hline ADAM10 & 14 vs. C & Down & 0.001 & WISP1 & 14 vs. C & Down & 0.001 \\
\hline CDKN1A & 14 vs. C & Down & 0.019 & WNT11 & 14 vs. C & Up & 0.018 \\
\hline CTNNB1 & 14 vs. C & Down & 0.013 & $\mathrm{~B} 2 \mathrm{M}$ & 14 vs. C & Down & 0.001 \\
\hline EP300 & 14 vs. C & Down & 0.001 & FOSL1 & 21 vs. C & Up & 0.013 \\
\hline FZD7 & 14 vs. C & Up & 0.013 & HES1 & 21 vs. C & $\mathrm{Up}$ & 0.043 \\
\hline HDAC1 & 14 vs. C & Down & 0.021 & SNW1 & 21 vs. C & Up & 0.007 \\
\hline H0XB4 & 14 vs. C & $\mathrm{Up}$ & 0.003 & WISP1 & 21 vs. C & $\mathrm{Up}$ & 0.038 \\
\hline JAG1 & 14 vs. C & Down & 0.016 & RPLP0 & 21 vs. C & Down & 0.047 \\
\hline JAG2 & 14 vs. C & Down & 0.006 & & & & \\
\hline
\end{tabular}

C, control group; Up, upregulated; Down, downregulated.

\section{Conclusions}

Overexpression of Lmx1 $\alpha$ and NTN promoted differentiation of h-BMSCs to action potential-producing DA neurons. In the process of differentiation, $\operatorname{Lmx} 1 \alpha$ and NTN may affect the expression of Notch signal-related genes. In addition, h-BMSCs undergo an intermediate step of differentiating into neural stem cells before differentiating into DA neurons. Based on these findings, $\operatorname{Lmx} 1 \alpha$ and NTN-expressing $\mathrm{h}$-BMSCs is a promising approach for cell therapy in PD.

\section{Data Availability}

The data used to support the findings of the study are included within the article.

\section{Conflicts of Interest}

The authors declare that they have no conflicts of interest.

\section{Authors' Contributions}

Jinhua Zhang and Bo Yang contributed equally to this work. Jinhua Zhang and Bo Yang wrote the article. Lilin Luo, Linhui $\mathrm{Li}$, and Xuantao Yang participated in data interpretation and critical revision of article. Juanjuan Zhang and Yuxin Xie contributed to the acquisition of reagents and materials. Jinhua Zhang, Bo Yang, Juanjuan Zhang, and Yuxin Xie executed the study. Wanpu Wang and Shuaiyao Lu designed the study. Wanpu Wang approved the study.

\section{Acknowledgments}

This work was supported by Yunnan Health Training Project of High Level Talents (H-2019001), Yunnan Fundamental Research Projects (202101AT070238), National Natural Science Foundation of China (81660238), and Yunnan Province Clinical Center for Hematologic Disease (2020LCZXKF-XY13).

\section{References}

[1] G. E. Alexander, “Biology of Parkinson's disease: pathogenesis and pathophysiology of a multisystem neurodegenerative disorder," Dialogues in Clinical Neuroscience, vol. 6, no. 3, pp. 259-280, 2004.

[2] L. Bertram and R. E. Tanzi, "The genetic epidemiology of neurodegenerative disease," Journal of Clinical Investigation, vol. 115, no. 6, pp. 1449-1457, 2005.

[3] D. Lo Furno, G. Mannino, and R. Giuffrida, "Functional role of mesenchymal stem cells in the treatment of chronic neurodegenerative diseases," Journal of Cellular Physiology, vol. 233, no. 5, pp. 3982-3999, 2018.

[4] W.-P. Wang, Z.-L. He, S.-Y. Lu et al., "Dopaminergic neuronlike cells derived from bone marrow mesenchymal stem cells by $\operatorname{Lmx} 1 \alpha$ and neurturin overexpression for autologous cytotherapy in hemiparkinsonian rhesus monkeys," Current Stem Cell Research \& Therapy, vol. 10, no. 2, pp. 109-120, 2015.

[5] T.-H. Wang, Z.-T. Feng, P. Wei, H. Li, Z.-J. Shi, and L.-Y. Li, "Effects of pcDNA3- $\beta$-NGF gene-modified BMSC on the rat model of Parkinson's disease," Journal of Molecular Neuroscience, vol. 35, no. 2, pp. 161-169, 2008.

[6] M. Ye, X.-J. Wang, Y.-H. Zhang et al., "Transplantation of bone marrow stromal cells containing the neurturin gene in rat model of Parkinson's disease," Brain Research, vol. 1142, pp. 206-216, 2007.

[7] V. V. Chizhikov and K. J. Millen, "Control of roof plate formation by Lmxla in the developing spinal cord," Development, vol. 131, no. 11, pp. 2693-2705, 2004.

[8] S. Chung, A. Leung, B.-S. Han et al., "Wnt1-lmx1a forms a novel autoregulatory loop and controls midbrain dopaminergic differentiation synergistically with the SHH-FoxA2 pathway," Cell Stem Cell, vol. 5, no. 6, pp. 646-658, 2009.

[9] M. O. Clements, A. Godfrey, J. Crossley, S. J. Wilson, Y. Takeuchi, and C. Boshoff, "Lentiviral manipulation of gene expression in human adult and embryonic stem cells," Tissue Engineering, vol. 12, no. 7, pp. 1741-1751, 2006.

[10] M. Bauer, J. Szulc, M. Meyer et al., "Delta-like 1 participates in the specification of ventral midbrain progenitor derived dopaminergic neurons," Journal of Neurochemistry, vol. 104, no. 4, pp. 1101-1115, 2008. 
[11] J. L. Ables, J. J. Breunig, A. J. Eisch, and P. Rakic, "Not (ch) just development: notch signalling in the adult brain," Nature Reviews Neuroscience, vol. 12, no. 5, pp. 269-283, 2011.

[12] J. Mahler, A. Filippi, and W. Driever, "DeltaA/DeltaD regulate multiple and temporally distinct phases of notch signaling during dopaminergic neurogenesis in zebrafish," Journal of Neuroscience, vol. 30, no. 49, pp. 16621-16635, 2010.

[13] Y. Song, J. R. Willer, P. C. Scherer et al., "Neural and synaptic defects in slytherin, a zebrafish model for human congenital disorders of glycosylation," PLoS One, vol. 5, no. 10, Article ID e13743, 2010.

[14] L. Wu and J. D. Griffin, "Modulation of notch signaling by mastermind-like (MAML) transcriptional co-activators and their involvement in tumorigenesis," Seminars in Cancer Biology, vol. 14, no. 5, pp. 348-356, 2004.

[15] C. P. Hodgkinson, J. A. Gomez, M. Mirotsou, and V. J. Dzau, "Genetic engineering of mesenchymal stem cells and its application in human disease therapy," Human Gene Therapy, vol. 21, no. 11, pp. 1513-1526, 2010.

[16] P. T. Kotzbauer, P. A. Lampe, R. O. Heuckeroth et al., "Neurturin, a relative of glial-cell-line-derived neurotrophic factor," Nature, vol. 384, no. 6608, pp. 467-470, 1996.

[17] J. Wu, C. Sheng, Z. Liu et al., "Lmxla enhances the effect of iNSCs in a PD model," Stem Cell Research, vol. 14, no. 1, pp. 1-9, 2015.

[18] H. Doucet-Beaupré, C. Gilbert, M. S. Profes et al., "Lmxla and Lmx $1 b$ regulate mitochondrial functions and survival of adult midbrain dopaminergic neurons," Proceedings of the National Academy of Sciences, vol. 113, no. 30, pp. E4387-E4396, 2016.

[19] S. Hu, M. Li, and R. Akkina, "Generation of high-titer pseudotyped lentiviral vectors," Methods in Molecular Biology, vol. 1937, pp. 125-134, 2019.

[20] K. Ma, L. Fox, G. Shi et al., "Generation of neural stem cell-like cells from bone marrow-derived human mesenchymal stem cells," Neurological Research, vol. 33, no. 10, pp. 1083-1093, 2011.

[21] Q. Bian, J. H. Huang, S. F. Liu et al., "Different molecular targets of icariin on bMSCs in CORT and OVX -rats," Frontiers in Bioscience, vol. E4, no. 1, pp. 1224-1236, 2012.

[22] R. A. Oldershaw, S. R. Tew, A. M. Russell et al., "Notch signaling through jagged-1 is necessary to initiate chondrogenesis in human bone marrow stromal cells but must be switched off to complete chondrogenesis," Stem Cells, vol. 26, no. 3, pp. 666-674, 2008.

[23] N. Sestan, S. Artavanis-Tsakonas, and P. Rakic, "Contactdependent inhibition of cortical neurite growth mediated by notch signaling," Science, vol. 286, no. 5440, pp. 741-746, 1999.

[24] S. Hitoshi, T. Alexson, V. Tropepe et al., "Notch pathway molecules are essential for the maintenance, but not the generation, of mammalian neural stem cells," Genes \& Development, vol. 16, no. 7, pp. 846-858, 2002.

[25] J. L. Salazar and S. Yamamoto, "Integration of drosophila and human genetics to understand notch signaling related diseases," Advances in Experimental Medicine and Biology, vol. 1066, pp. 141-185, 2018.

[26] F. N. Velazquez, B. L. Caputto, and F. D. Boussin, "c-Fos importance for brain development," Aging, vol. 7, no. 12, pp. 1028-1029, 2015.

[27] S. Ankam, C. K. Lim, and E. K. F. Yim, "Actomyosin contractility plays a role in MAP2 expression during nanotopography-directed neuronal differentiation of human embryonic stem cells," Biomaterials, vol. 47, pp. 20-28, 2015.

[28] P. Gruss and C. Walther, "Pax in development," Cell, vol. 69, no. 5, pp. 719-722, 1992. 